\title{
Application of the Parametrical Surface Wave Prediction Model to Rapidly Varying Wind Fields During JONSWAP 1973
}

\author{
H. GÜNTHER AND W. ROSENTHAL
}

\author{
Institut für Geophysik. Universität Hamburg, and \\ Max-Planck Institut für Meteorologie, Hamburg, Germany

\section{K. RiCHTER} \\ Deutsches Hydrographisches Institut, Hamburg. Germany
}

\begin{abstract}
The capability of a parametrical surface wave model to predict the sea state on a small array for highly variable wind fields is shown for three examples. The model treats the wind sea, for which the nonlinear interaction is most effective, in a parametrical sense. The swell is propagated along characteristics, and the source function for the swell is assumed to be zero. The model output is compared with wave measurements from the JONSWAP 73 experiment.
\end{abstract}

\section{INTRODUCTION}

This paper is the third in a series of related papers. The first paper (Günther et al. [1979], henceforth referred to as Paper I) gives a general description of the hybrid parametric wave prediction model which is based on the parametrical wind-sea model described by Hasselmann et al. [1976]. The second paper [Ewing et al., 1979] considers the validation of the hybrid model on a large scale and its application to calculate extreme value wave statistics for the North Sea from a set of $\mathbf{4 0}$ storms selected from the last decade. This paper is concerned with the small scale capabilities of the model in highly nonstationary cases to test its performance under rather limiting conditions.

For this study we use environmental data gathered during JONSWAP 73 in the North Sea west of the Island of Sylt, using essentially the same station array as described by Hasselmann et al. [1973]. Although for such a relatively small region the input value of the wave spectrum along the boundaries of the area have generally had a rather strong influence on the form of the wave field within the region, for strongly variable wind fields the spectral development within the prediction area was still sufficiently large to provide a meaningful test of the model capability.

\section{Structure of the Model}

As described in more detail in paper I, the spectrum is divided into a swell region, in which nonlinear interactions are insignificant, and a wind-sea region, in which the energy balance is strongly controlled by nonlinear transfer.

\section{Wind-Sea Domain}

The standard energy transport equation in water of infinite depth reads

$$
\frac{\partial F}{\partial t}+\mathbf{v} \nabla F=T
$$

where $F=F(\mathbf{r}, f, \theta)$ is the two-dimensional energy density spectrum with respect to frequency $f$ (in Herz) and direction $\theta$ (in radians); $\mathbf{r}$ is location; $v=v(f, \theta)$, group velocity; and $T$ is the source function. Equation (1) is first transformed into an equivalent equation in parameter space:

Copyright $\odot 1979$ by the American Geophysical Union.

$$
\frac{\partial a_{i}}{\partial t}+D_{i j k} \frac{\partial a_{j}}{\partial x_{k}}=S_{i}
$$

where in our case the parameters chosen are the JONSWAP parameters:

$$
\begin{aligned}
& a_{1}=f_{m}=\text { peak frequency; } \\
& a_{2}=\alpha=\text { Phillips constant; } \\
& a_{3}=\gamma=\text { peak enhancement factor; } \\
& a_{4}=\sigma_{a}=\text { peak width at low frequency side; } \\
& a_{5}=\sigma_{b}=\text { peak width at high frequency side. }
\end{aligned}
$$

The transformation method as well as the resultant transport coefficients $D_{l j k}$ and source functions $S_{l}$ are given in paper I, so that we may restrict ourselves here to a short review.

In the papers of Hasselmann et al. [1973, 1976] it is shown that growing one-dimensional wind-sea spectra $E(f)$ can be described by a class of functions specified by the five parameters listed above:

$$
\begin{aligned}
E(f)=\alpha g^{2}(2 \pi)^{-4} f^{-5} \exp \left[-\frac{5}{4}\left(\frac{f}{f_{m}}\right)^{-4}\right. & +\ln \gamma \exp \\
& \left.\left(-\frac{\left(f-f_{m}\right)^{2}}{2 \sigma^{2} f_{m}^{2}}\right)\right]
\end{aligned}
$$

where

$$
\begin{array}{ll}
\sigma=\sigma_{a} & f \leq f_{m} \\
\sigma=\sigma_{b} & f>f_{m}
\end{array}
$$

(see Figure 1). The two-dimensional energy spectrum $F(f, \theta)$ was constructed from (3) by assuming a fixed cosine square spreading factor defined relative to the local wind direction $\theta_{0}$ :

$$
\begin{gathered}
F(f, \theta)=E(f) \frac{2}{\pi} \cos ^{2}\left(\theta-\theta_{0}\right) \quad\left|\left(\theta-\theta_{0}\right)\right| \leq \frac{\pi}{2} \\
F(f, \theta)=0 \quad\left|\left(\theta-\theta_{0}\right)\right|>\frac{\pi}{2}
\end{gathered}
$$

We note that the directional distribution is not treated prognostically, and that the parameter set $a_{1}, \cdots, a_{b}$ accordingly includes no directional parameters. This approximation can be justified for wind felds which are not too rapidly varying [cf. Hasselmann et al., 1976] but is a shortcoming which may be 


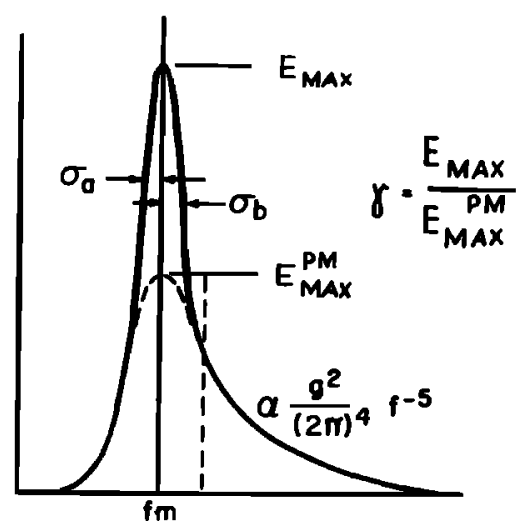

Fig. 1. Definition of JONSWAP parameters.

expected to (and indeed will) reveal itself in the present application of the model to rapidly changing wind fields. As a next step, however, it is planned to extend the model (using, a mong other analyses, the results of the present investigation) to include prognostic directional parameters.

The numerical procedure used to solve (2) is a predictorcorrector scheme originally described by McCormack, but modified for our more complicated system of equations. Introducing the parameter and source function vectors

$$
A=a_{1}, a_{2}, a_{3}, a_{4}, a_{5} \quad C=S_{1}, S_{2}, S_{3}, S_{4}, S_{6}
$$

and the two coefficient matrices

$$
B_{1}=\left(D_{t, j, 1}\right) \quad B_{2}=\left(D_{i, j, 2}\right)
$$

(2) may be written

$$
\frac{\partial A}{\partial t}+B_{1} \frac{\partial A}{\partial x_{1}}+B_{2} \frac{\partial A}{\partial x_{2}}=C
$$

Let $\Delta_{1}{ }^{-}, \Delta_{2}{ }^{-}$and $\Delta_{1}{ }^{+}, \Delta_{2}{ }^{+}$denote upwind and downwind difference operators, respectively, in the coordinate directions $x_{1}$ and $x_{2}$ :

$$
\begin{array}{rr}
\Delta_{1}-A=\left(A_{l, k}-A_{l-\epsilon, k}\right) \epsilon & \Delta_{2}-A=\left(A_{l, k} A_{l, k-\mu}\right) \mu \\
\Delta_{1}{ }^{+} A=\left(A_{l+\epsilon, k}-A_{l, k}\right) \epsilon & \Delta_{2}{ }^{+} A=\left(A_{l, k+\mu}-A_{l, k}\right) \mu
\end{array}
$$

where the indices $l, k$ denote grid points and $\epsilon$ and $\mu$ take the values \pm 1 , depending on the sign of the associated wind component.

The value $A^{n+1}$ is first predicted by

$$
\tilde{A}^{n+1}=A^{n}-\frac{\Delta t}{\Delta x}\left\{B_{1}^{n} \Delta_{1}^{-}+B_{2}^{n} \Delta_{2}^{-}\right\} A^{n}+\Delta t C^{n}
$$

(predictor). From this value, $\tilde{A}^{n+1}$, coefficients $\tilde{B}_{1}^{n+1}, \tilde{B}_{2}^{n+1}$ and the source field $C^{n+1}$ are calculated. Finally, the corrected field $A^{n+1}$ is determined by

$$
\begin{aligned}
A^{n+1}= & \frac{1}{2}\left(\tilde{A}^{n+1}+\tilde{A}^{n}\right)-\frac{1}{2} \frac{\Delta t}{\Delta x} \\
& \left\{\tilde{B}_{1}^{n+1} \Delta_{1}{ }^{+}+\tilde{B}_{2}^{n+1} \Delta_{2}{ }^{+}\right\} \tilde{A}^{n+1}+\frac{1}{2} \Delta t \tilde{C}^{n+1}
\end{aligned}
$$

(corrector). The use of upwind (downwind) differences in the predictor (corrector) fields ensures that only the boundary values associated with incoming parameter fields affect the predictor equation.

The scheme is of second order in space and time, and the stability criterion of the corresponding homogeneous equation with a constant coefficient matrix is

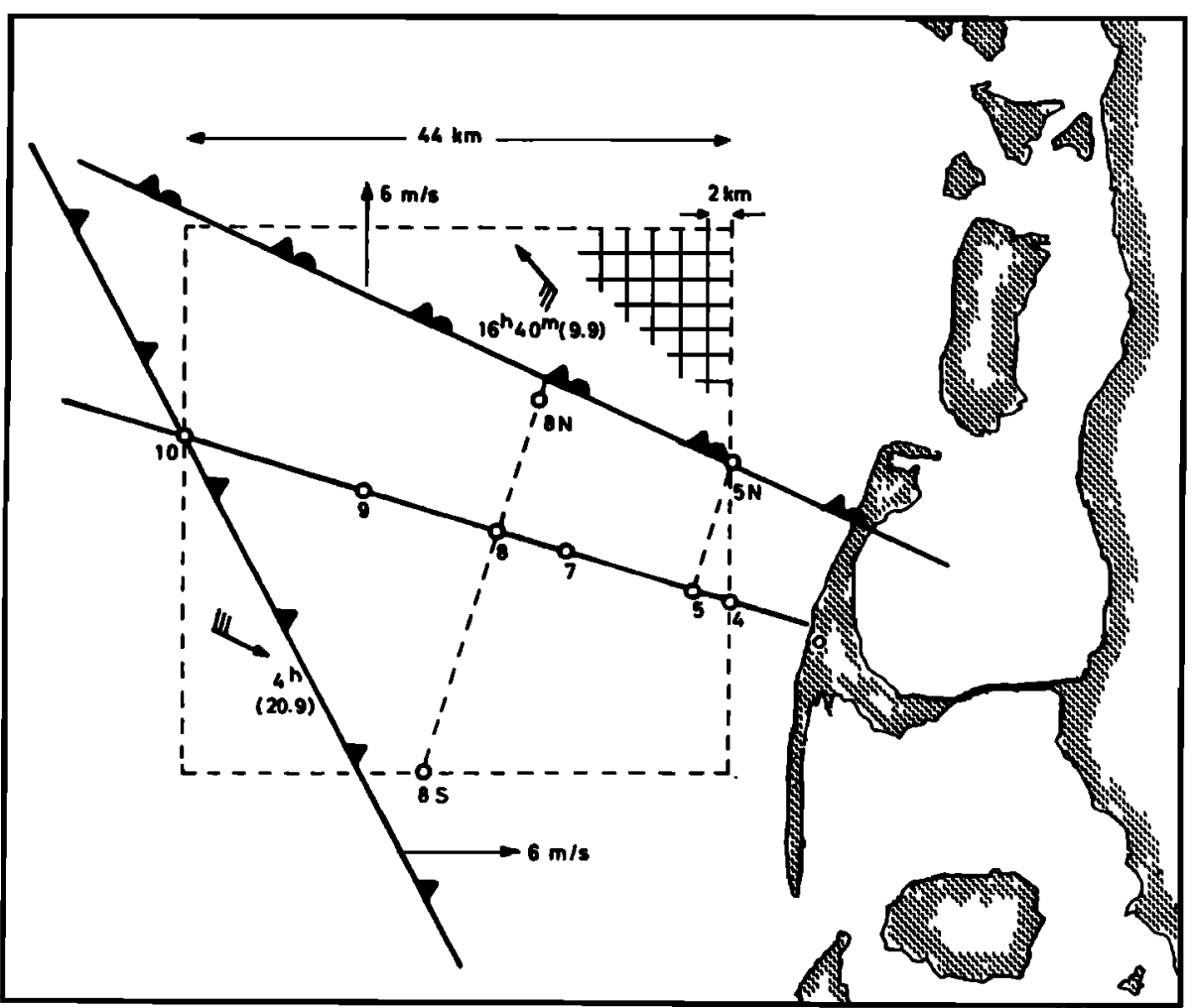

Fig. 2. Hindcast site, grid pattern, and meteorological situation during hindcast period A. Note that the positions of the two fronts correspond to two different times. Arrows indicate frontal velocities. Circles indicate stations of the JONSWAP array. 

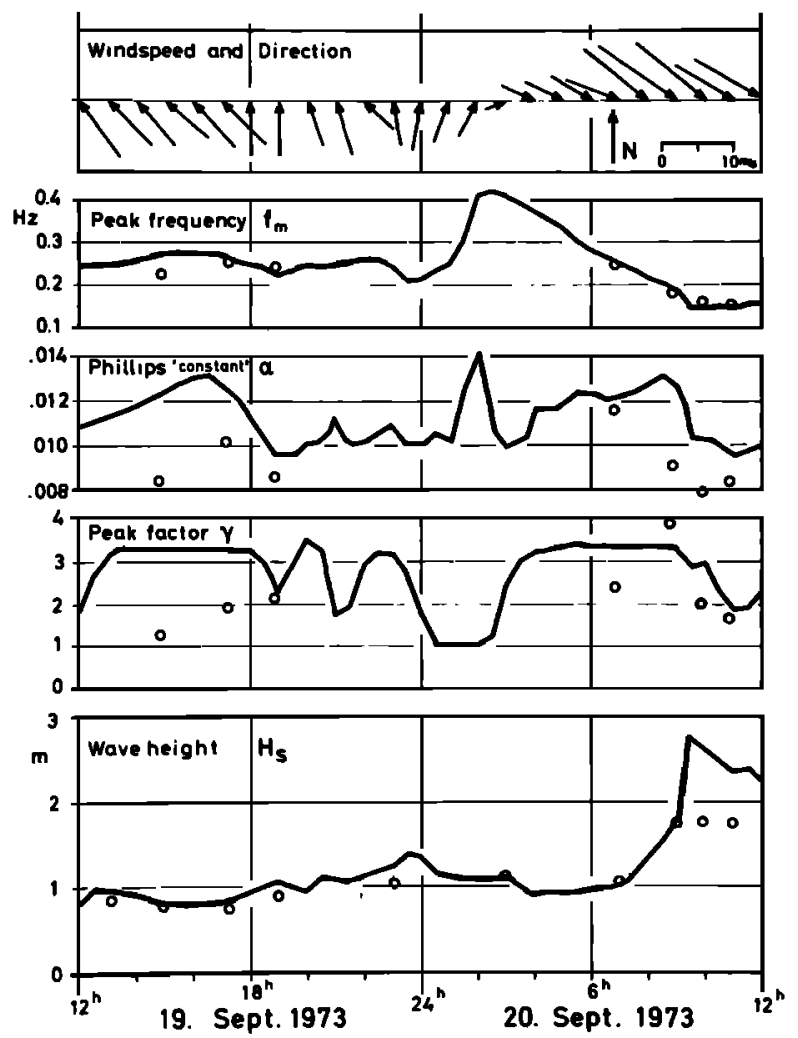

JONSWAP 73, Station 7

Fig. 3. Time series of hindcast A for station 7. Solid line represents hindcast; circles represent measurements.

$$
\frac{\Delta x}{\Delta t} \geq D_{\max }
$$

where $D_{\max }$ is the largest eigenvalue of the coefficient matrix. The condition requires that the ratio of grid spacing to time step $\Delta x / \Delta t$ be larger than the largest group velocity of the waves encountered in the prediction area.

For downwind boundaries (wind blowing out of the area) the corrector equation cannot be used, since there exist no adjacent grid points in the downwind direction. Therefore we set

$$
A^{n+1}=A^{n+1}
$$

at those points.

At the upwind boundaries, (9) and (10) are replaced by the prescribed boundary values $A^{n+1}$ of the incoming waves.

\section{Swell Domain}

For swell, the wind induced part of the source function (in (1)) vanishes, and the nonlinear interaction can be neglected (by definition). As in our case studies, the waves are treated as deep water waves; we also neglect dissipation of swell. Thus the right-hand side of (1) is zero, and the wave energy for different frequencies and directions propagates unattenuated along characteristics which, in our case, are straight lines. We have accordingly adopted a characteristic representation of the swell domain [cf. Barnett et al., 1969]. The transfer of energy from the cartesian to the characteristic grid can be managed by several procedures. In order to extend the model later to shallow water and therefore to curved characteristics we adopted the simple method described briefly in the following.
The space region covered by the Cartesian and also by the characteristic grid is divided into conveniently shaped space elements of finite size. Each space element covers at least one characteristic grid point per direction and one Cartesian gridpoint. The energy exchange between the two grids is carried out, when required, for each space element separately. The transfer occurs at each time step. The model includes a transfer of energy from wind-sea to swell and back. Details of this transfer are described in paper $I$.

\section{Measurements}

The wave and wind measurements used for the model were selected from the data set obtained during the JONSWAP 73 experiment. Surface wave measurements were made with wave riders and pitch-roll buoys at a sampling rate of $2 \mathrm{~Hz}$. Power spectra were computed as averages of the individual spectra of 10 successive time series pieces of 128-s duration by the FFTtechnique. Directional parameters were computed from the pitch-roll data by the technique described by Cartwright [1961] and Hasselmann et al. [1973]. The peak frequency $f_{m}$ of the wind sea was estimated by fitting a parabola to the three highest spectral values of the wind sea part of the spectrum. The significant wave height $H_{s}$ was taken as 4 times the rms surface displacement, i.e., as the square root of the integral of the power spectrum [cf. Kinsman, 1965].

\section{Model Parameters, Boundary, and INITIAL VALUES}

As prediction area, a $44 \times 44 \mathrm{~km}^{2}$ square within the JONSWAP array [Hasselmann et al., 1973] was chosen, in which observed spectra were available with high temporal and spatial
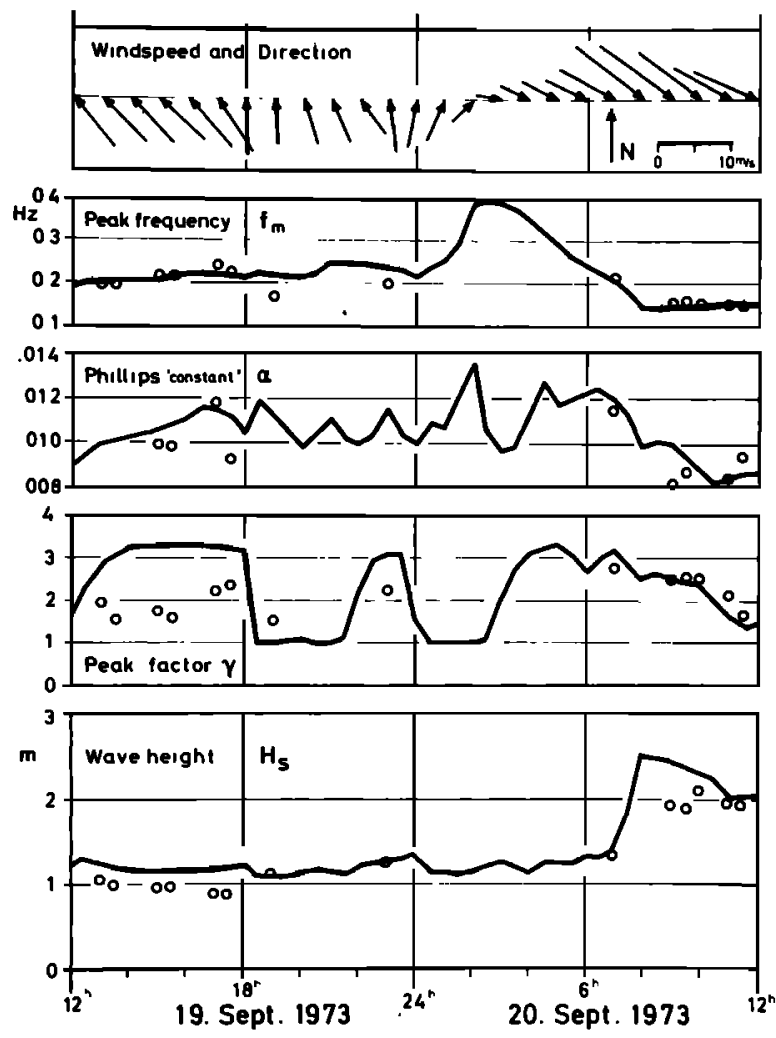

JONSWAP 73, Station 9

Fig. 4. Time series of hindcast A for station 9. Solid line represents hindcast; circles represent measurements. 

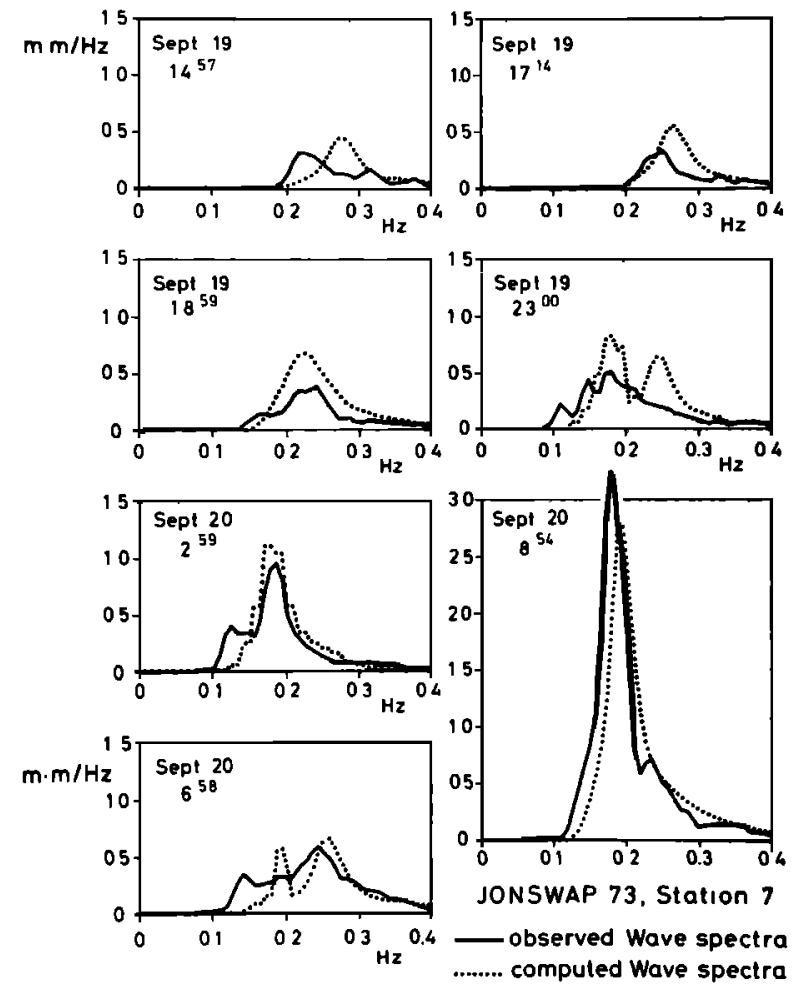

Fig. 5. Spectra at station 7 of hindcast A. Solid line represents measurements; dotted line represents hindcast.

density (Figure 2). The grid resolution of the model was $2 \mathrm{~km}$, the integration time step $5 \mathrm{~min}$. The swell propagation routine contained 24 direction bins of $15^{\circ}$ width and 24 frequency bands each of bandwidth $2 / 128 \mathrm{~Hz}$, covering the range 0.078 $0.45 \mathrm{~Hz}$.

\section{Boundary Values for the Wind Sea}

Boundary values on the eastern boundary, needed for winds with a positive westerly component, were calculated using fetch laws [Hasselmann et al., 1973]. For winds blowing into the array across the southern and western boundary the required boundary values for the wind sea were obtained from the measurements at stations $8 \mathrm{~S}$ and 10 , respectively (Figure 2 ). For winds blowing to the NW the upwind boundary values were obtained by interpolating between the measured values at station $8 \mathrm{~S}$ and the fetch-derived spectra at the SE corner. For winds blowing toward SW we used for the northern boundary an interpolation between the measured values at station 10 and the fetch law derived boundary values at the NE corner of the array. For winds blowing toward SE we inserted the measurements at station 10 for the entire northern boundary.

\section{Boundary Values for Swell}

Frequently, swell entered the array from the NW or SW directions. The appropriate boundary values were also obtained from stations $8 \mathrm{~S}$ and 10 . As shown later, swell energy entering over the northern border and appearing at the easterly stations appears to have been considerably overestimated by considering it equal to the station 10 values. This is probably due to the neglect of bottom dissipation processes, which is not permissible for longer period swell in this area [cf. Hasselmann et al., 1973].

\section{Initial Values}

The initial values for the wind-sea part were obtained by spatial interpolation from the station measurements. For the swell energy we assumed zero energy over the entire interior region of the array. Hindcast and observation therefore differed in the swell region of the spectrum during the first hours of the integration, before the swell had propagated into the region from the boundaries.

\section{THE WIND FiELD}

The wind field analysis for the three test cases was kindly provided by D. Heinricy from the Max-Planck-Institute for Meteorology in Hamburg, using data from surrounding weather stations of the German Weather Service and the surface winds-measured continuously at the JONSWAP land station and at stations 8 and 10 of the JONSWAP array.

\section{Hindcast A, September 19, 12:00 Hours to September 20, 12:00 HoURs}

\section{Wind Situation Over the German Bight}

Throughout most of the day on September 19 the German Bight experienced moderate southeasterly winds (Figure 3 ).

At night the frontal system depicted in Figure 2 crossed the JONSWAP array. After the arrival of an occluded front in the evening, the wind slowed down and shifted toward southerly directions. In the morning of September 20 a rather strong front with a pronounced shift in wind direction crossed the area from SSW to WNW. Behind the front the wind speed increased sharply to $12-15 \mathrm{~m} \mathrm{~s}^{-1}$.

To make best use of the detailed surface wind measurements (10-min averages each hour) available from three stations of
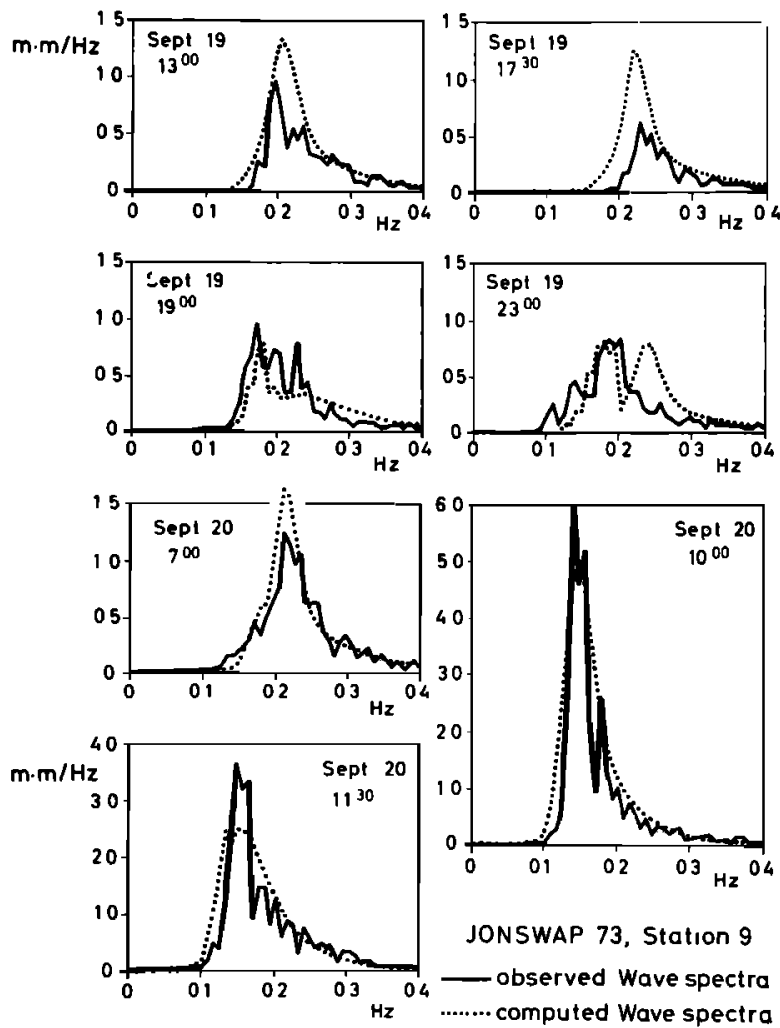

Fig. 6. Spectra at station 9 of hindcast A. Solid line represents measurements; dotted line represents hindcast. 

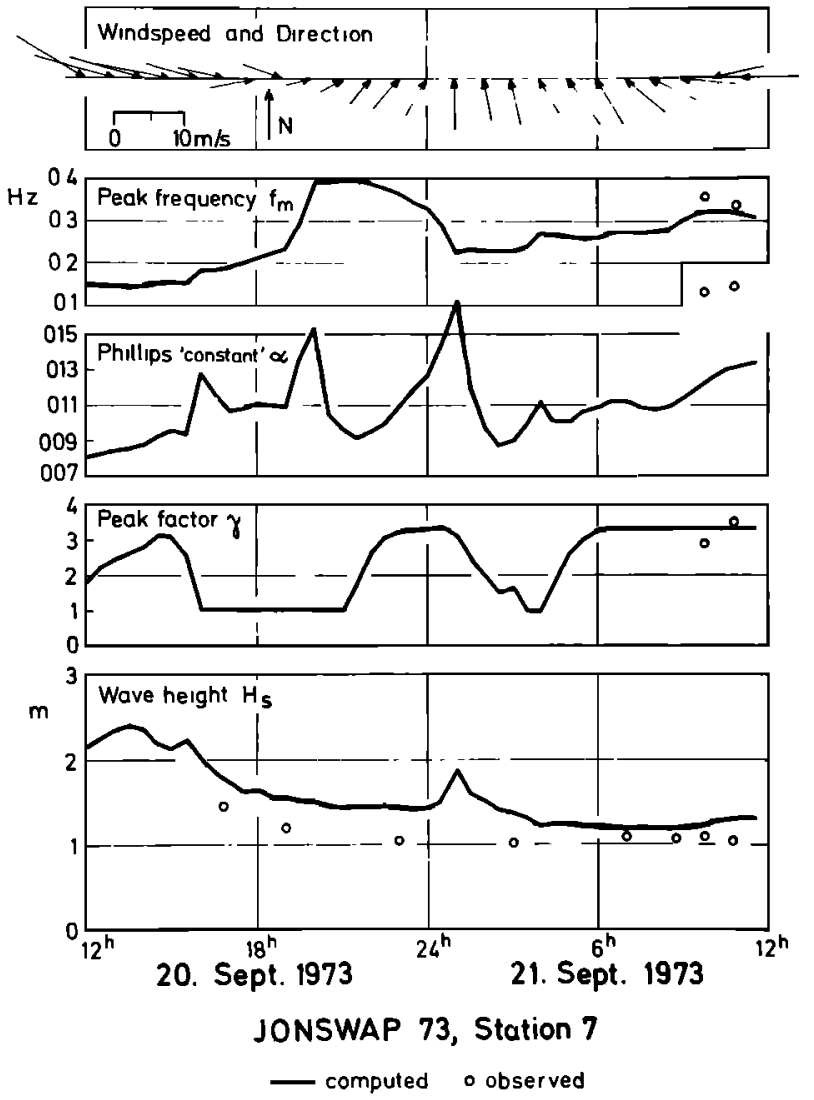

Fig. 7. Time series of hindcast B for station 7. Solid line represents hindcast; circles represent measurements.

the array, the following procedure was adopted to determine the wind vector at the model grid points:

Station 8 winds measured from a wind buoy [see Brümmer et al., 1974] were regarded as the most reliable for modeling wind variation over the area. Therefore hourly measurements at station 8 were propagated with the speed of the frontal system across the area, assuming that the variations are propagated with large-scale motion. Perpendicular to this base line the wind field was deduced under the restrictions of vanishing horizontal divergence and minimal wind vector deviation from the base line values. The validity of this procedure was verified by comparing with the other two continuous wind measurements.

\section{Hindcast Results}

The hindcast results were compared with measurements at stations 7 and 9, since these stations lie near the center of the area and thus have the largest distance to all boundaries.

Figures 3 and 4 show the time series of the wind field at stations 7 and 9 , the measured hindcasted time series of the wind-sea parameters $f_{m}, \alpha, \gamma$, and the significant wave height $H_{s}$ (for the complete spectrum). In the presence of broad band swell, determination of the wind-sea parameters from the observed spectra was not always possible, since a unique windsea peak frequency could not always be identified in the superimposed wind-sea and swell spectra. Thus no observed parameters are shown in the figures for these periods. The observed and hindcast spectra at stations 7 and 9 for various times of the hindcast are shown in Figures 5 and 6.

Comparing Figures 3 and 4, we see that during the period
September 19, 12-18 hours, the wind-sea parameters $f_{m}, \alpha$ are hindcasted better at station 9 than at station 7 , although the general time evolution is also well reproduced by the model at station 7 . This can be attributed to the stronger influence at station 7 of the initial values and of southern boundary values, which were obtained by interpolation of the values at station $8 \mathrm{~S}$ and the calculated fetch-limited values at the eastern boundary. The error in the hindcast indicates that the effective fetch was larger than assumed.

Because of lower windspeeds in the following period, September 19, 18:00 hours to September 20, 06:00 hours, most of the energy of the wave spectrum lies at frequencies less than the Pierson Moskowitz frequency, and is thus treated by the swell part of the model. Here only a comparison of $H_{s}$ is meaningful; this is good for both stations considered.

The last hindcast period, September 20, 06:00-12:00 hours, is governed by stronger winds from the NW. The peak frequency is well reproduced by the model, but $\alpha$ and $H_{s}$ are overpredicted at station 7 . This is probably due to shallowwater effects, which affect station 7 more than station 9 . The wavelength corresponding to the measured peak frequency is about $70 \mathrm{~m}$, and the water depth at station 7 and in the region to the NW, from which the waves originate, is about $14 \mathrm{~m}$. Thus bottom dissipation, not included in the present model, can probably not be neglected.

Comparison of observed and hindcast spectra is satisfactory at both stations 7 and 9. Occasional overprediction of the wind sea is probably due to the crude directional model we have used. This comes out more clearly in the next test cases and will be discussed there.
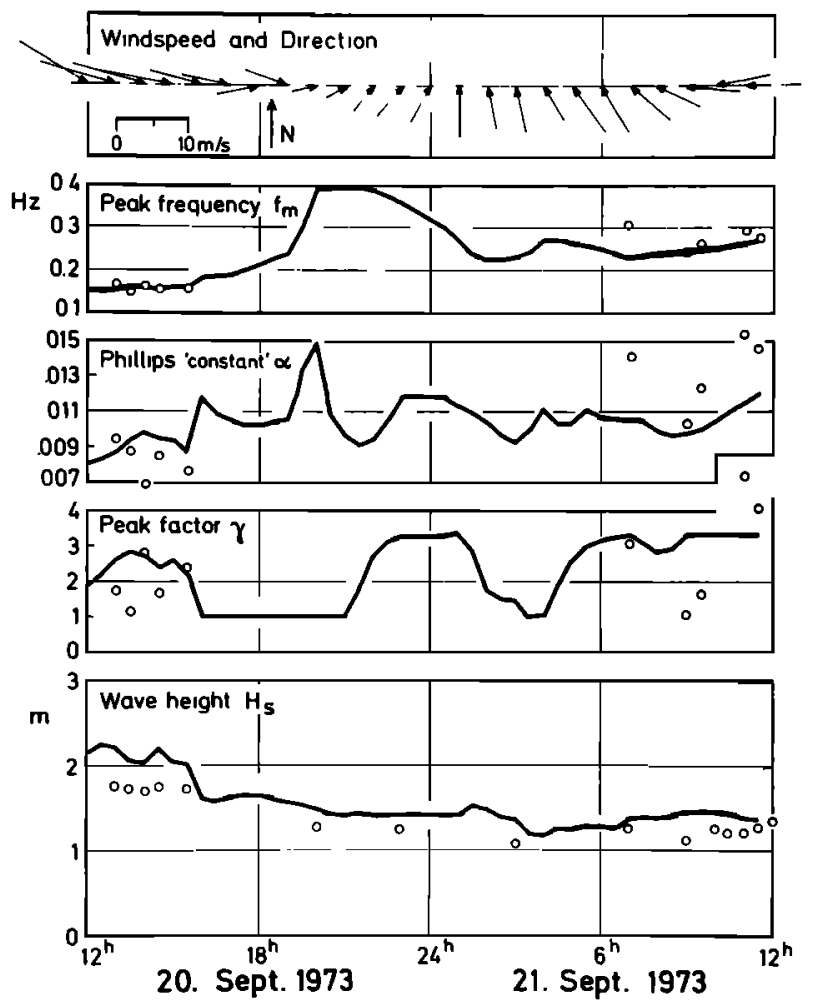

JONSWAP 73, Station 9

— computed o observed

Fig. 8. Time series of hindcast $B$ for station 9 . Solid line represents hindcast; circles represent measurements. 

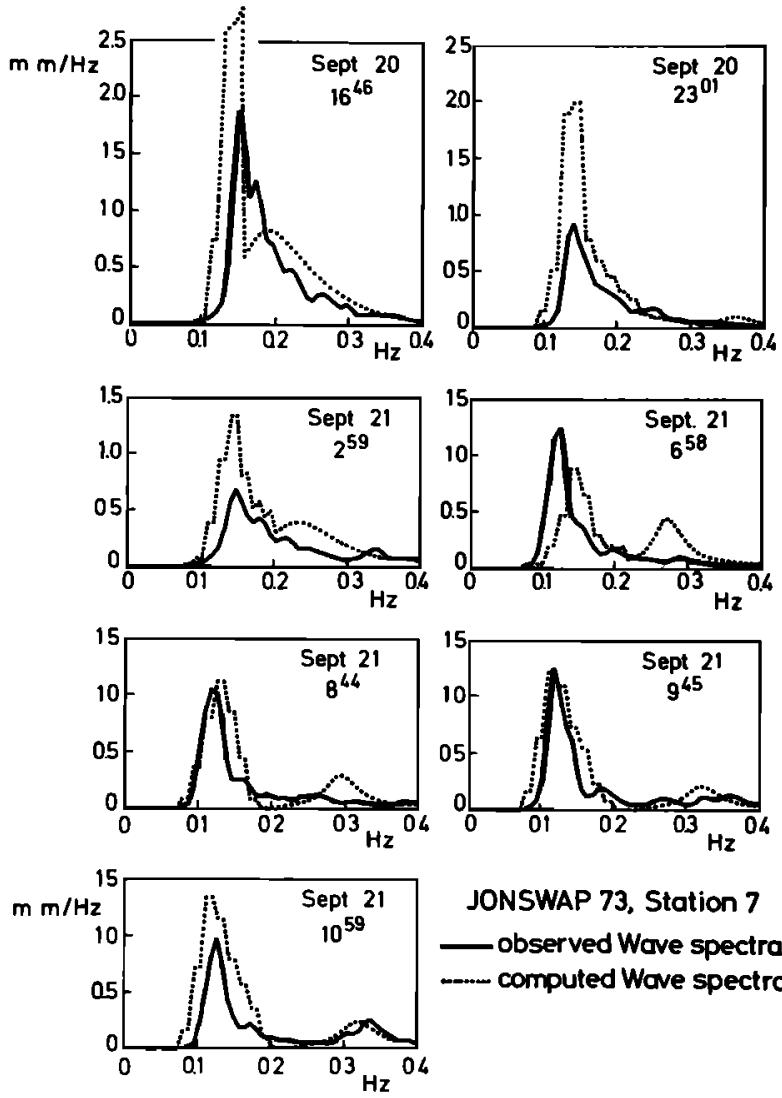

JONSWAP 73, Station 7

- observed Wove spectra -...- computed Wave spectra

Fig. 9. Spectra at station 7 of hindcast B. Solid line represents measurements; dotted line represents hindcast.
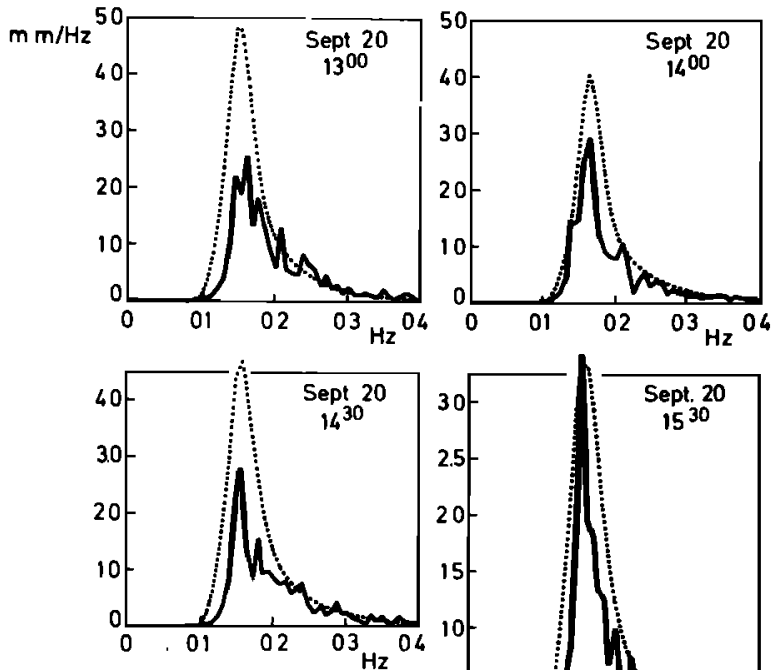

$\mathrm{m} \cdot \mathrm{m} / \mathrm{Hz}$

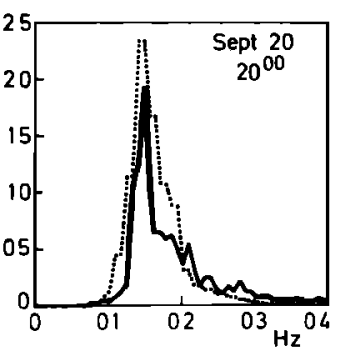

JONSWAP 73, Station 9

- observed Wave spectra ...-...... computed Wave spectro

Fig. 10. Spectra at station 9 of hindcast B (September 20). Solid line represents measurements; dotted line represents hindcast.
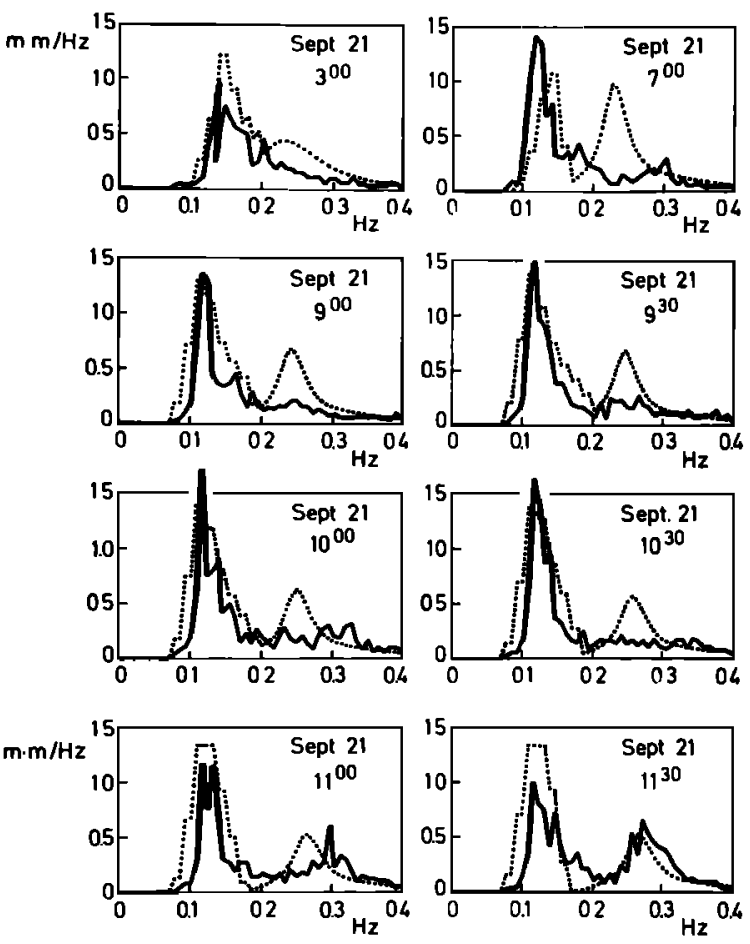

JONSWAP 73, Station 9

- observed Wave spectra

...-.-compuled Wave spectra

Fig. 11. Spectra at station 9 of hindcast B (September 21). Solid line represents measurements; dotted line represents hindcast.
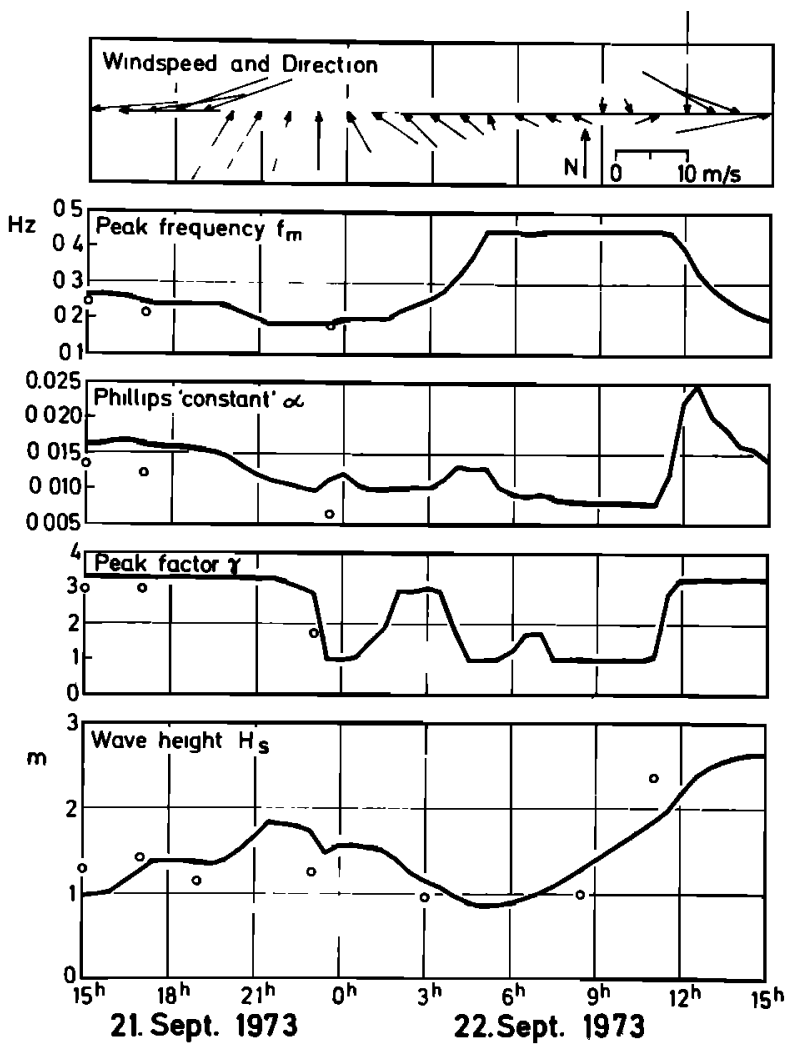

JONSWAP 73, Station 7

- computed o observed

Fig. 12. Time series of hindcast $\mathrm{C}$ for station 7 . Solid line represents hindcast; circles represent measurements. 

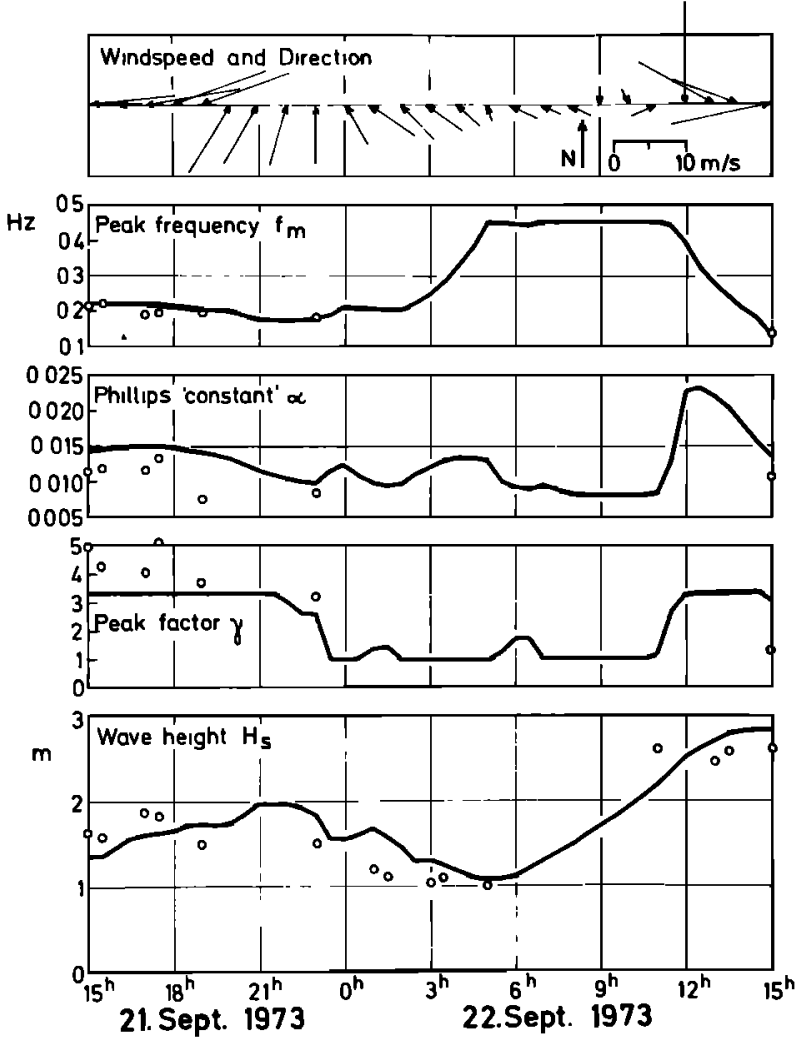

JONSWAP 73, Station 9

- computed o observed

Fig. 13. Time series of hindcast $\mathbf{C}$ for station 9. Solid line represents hindcast; Circles represent measurements.

\section{Hindcast B, September 20, 12:00 Hours to SEPTEMBER 21, 12:00 Hours}

\section{Wind Situation Over the German Bight}

Gradually decreasing WNW winds prevailed during the afternoon of September 20. In the evening a large-scale wind gradient region crossed the area, so that the wind field could be modeled as homogeneous over the hindcast area, the time evolution at station 8 simply being extended to the whole area (see the upper panels of Figures 7 and 8).

\section{Hindcast Results}

During most of the period, non dispersed swell governs the wave spectrum, so that the measured wave spectra do not provide clearly defined wind-sea parameters with which to compare the model. However, wave height is well modeled, although always slightly overestimated.

Comparing the spectra (Figures 9-11), the energies at low frequencies again show better agreement at station 9 than at station 7 . As before, we attribute this to energy dissipation due to bottom effects in shallow water. A conspicuous feature is the overprediction of high-frequency energies (for instance from 03:00 to 10:30 hours of September 21 at station 9). This can be attributed to the assumption of the model that the mean wave direction of the wind sea coincides always with the instantaneous wind direction. This approximation is based on the concept of a very rapid redistribution of wave energy by nonlinear wave-wave interaction, and must break down for rapidly turning winds [cf. Hasselmann, 1978]. In the present case, the wave direction changes by $130^{\circ}$ at $0.4-\mathrm{Hz}$ frequency during the period in question, as shown by the pitch-roll measurements at station 10 , and lags the change in wind direction significantly. Methods of improving the model in regard to this shortcoming will be discussed briefly in section 10.

\section{Hindcast C, September 21, 15:00 Hours to SEPTEMBER 22, 15:00 Hours}

\section{Wind Situation}

East to northeasterly winds increased to about $15 \mathrm{~m} \mathrm{~s}^{-1}$ by the evening of September 21. After passage of a warm front, the wind shifted to SE and decreased. In the morning of September 22 a slowly progressing cold front crossed the array from SW. It caused an abrupt wind shift to a westerly direction and a sharp increase in the wind speed to about $15 \mathrm{~m} \mathrm{~s}^{-1}$.

During the actual frontal passages, a very complicated wind pattern occurred across the area. We feel unable to reconstruct it in detail from the three wind time series of the array, and have assumed simply, as in Hindcast B, that the wind measured at station 8 is representative of the entire area. The upper panels of Figures 12 and 13 show the time series of the wind as assumed over the entire hindcast area.

\section{Hindcast results}

Figures 12 and 13 show good agreement for hindcast and measured wind sea and swell parameters as well as for wave height. The hindcast spectra (Figures 14 and 15) at stations 7 and 9 at 15:00 hours do not show the swell because it was not included in the initial field. The same holds for the spectrum at 15:30 hours at station 7 . The time of sharp wind shift between
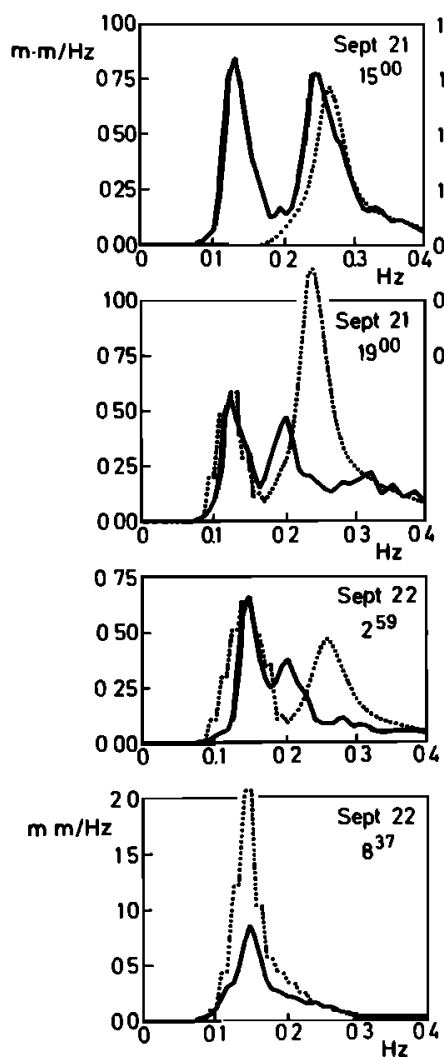

JONSWAP 73, Station? - observed Wave spectro -.-.-- computed Wave spectra
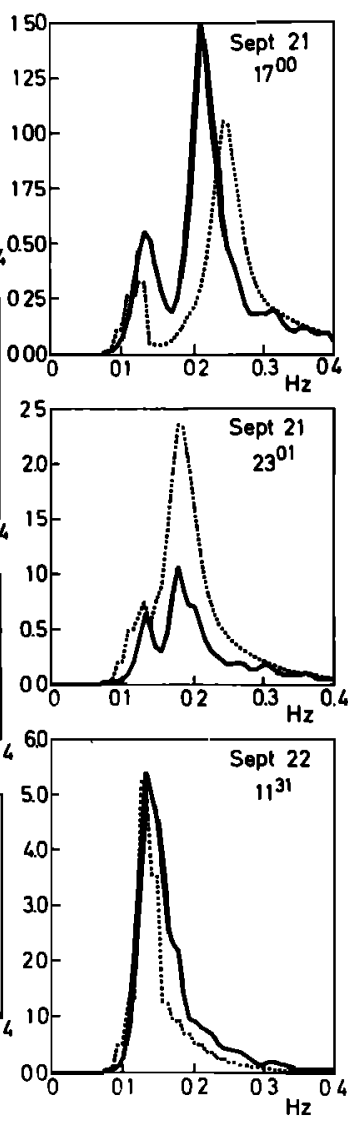

Fig. 14. Spectra at station 7 of hindcast C. Solid line represents measurements; Dotted line represents hindcast. 


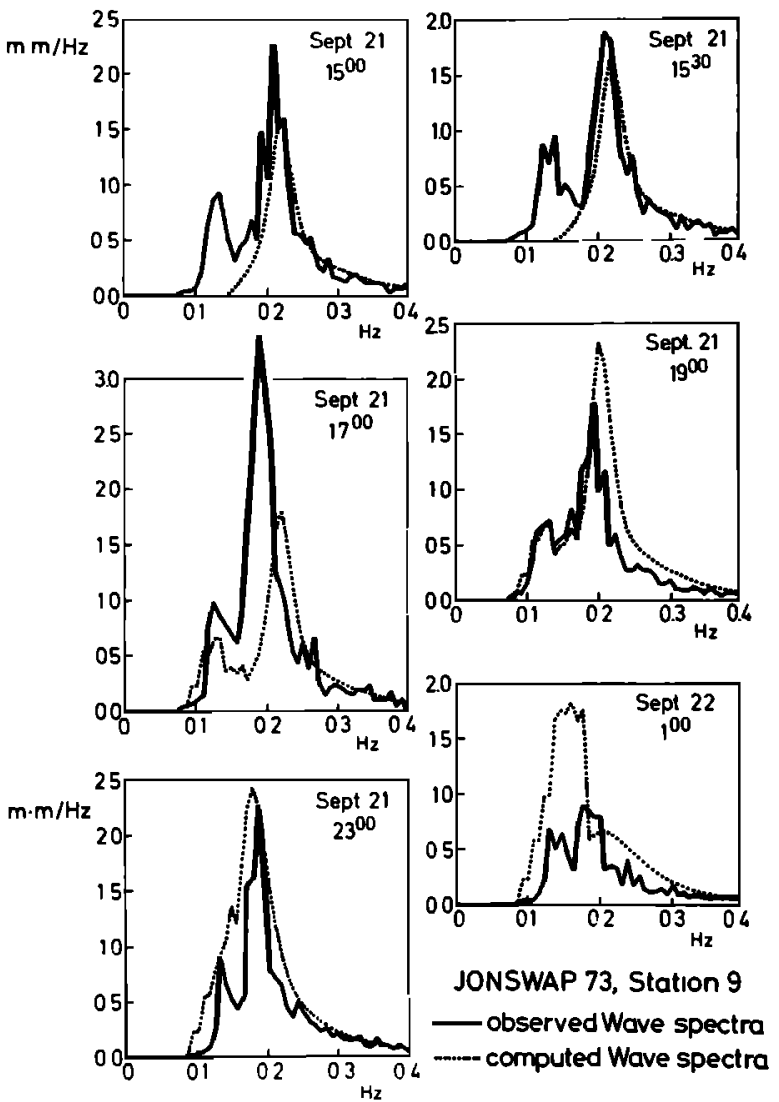

Fig. 15. Spectra at station 9 for the first half of hindcast $C$ period. Solid line represents measurements; dotted line represents hindcast.

17:00 and 19:00 hours shows differences between hindcast and measured spectra. However, as already mentioned, we could not resolve the passage of the warm front with sufficient accuracy to define a reliable wind field during that time.

Subsequent spectra show an overestimation of the wave energy by the model, especially at station 7. Again, this is presumably caused by the oversimplified directional response discussed earlier. A contributing cause to the overestimation at station 7 could also be bottom friction, since the long waves coming from the south cross shallow water in the southern part of the hindcast area; this effect persists until the wind and wind sea become more westerly in the latter part of the hindcast period.

This is supported by the spectra for the deeper station 9 , which show good agreement between model and measurement after September 22, 03:30 hours (Figure 16).

\section{INFLUENCE OF THE BOUNDARY VALUES}

As described in section 4, measured spectra enter the model as boundary value input along the upwind boundary of the region. Although this is necessary for a small array with open boundaries, it raises the question whether there is any significant influence of the source function on the results or whether the model merely propagates wave energy into the interior from the boundary with the group velocity of the appropriate wave components. In considering this question it is important to note the hybrid structure of the model. While the swell part operates in a simply propagating mode, the wind-sea part, by definition, cannot exist without a source term. To show the difference between pure energy propagation from the bounda-
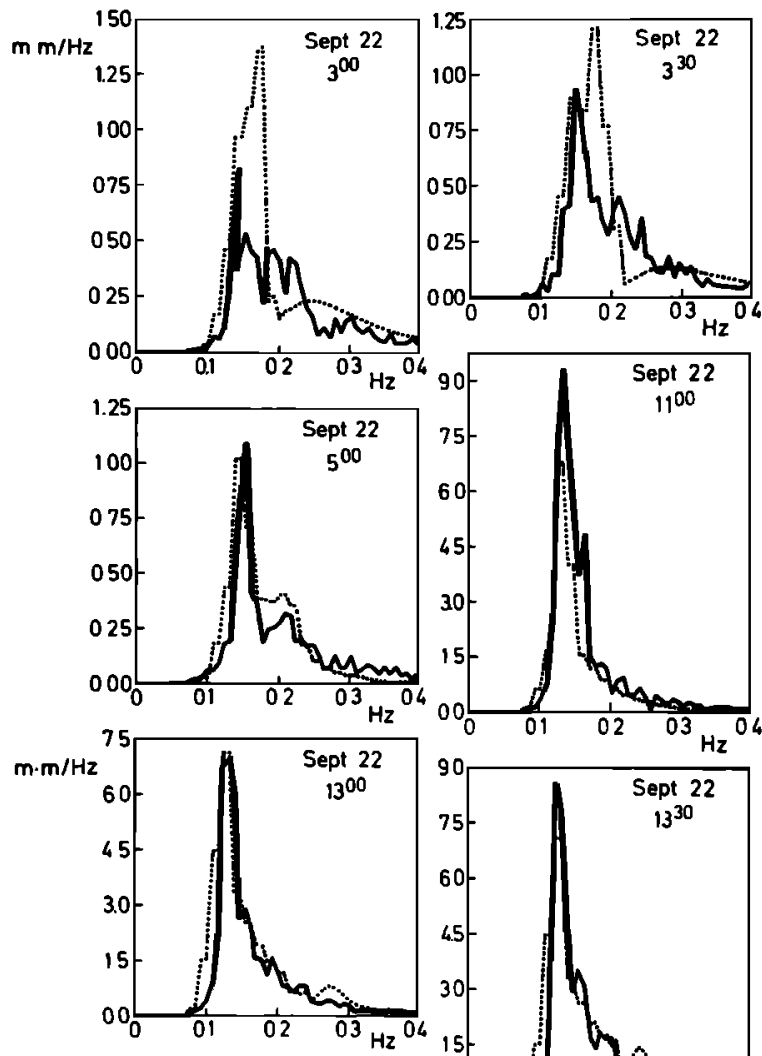

JONSWAP 73, Station 9 - observed Wave spectra

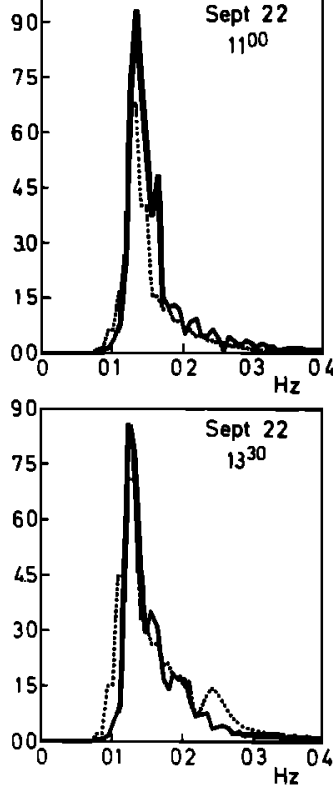

Fig. 16. Spectra at station 9 for the' second half of hindcast $\mathrm{C}$ period. Solid line represents measurements; dotted line represents hindcast.

ries and the complete prediction including generation within the region, the model was run with a vanishing wind field (to include a wind-sea component, the wind was actually not set identical to zero, but was restricted to stay below $3 \mathrm{~m} \mathrm{~s}^{-1}$ ). As to be expected, for the swell frequencies there is no difference between the zero and nonzero wind calculations, and one finds only minor changes of the significant wave height in swelldominated situations. Figure 17 shows the significant wave height calculations for the zero-wind case together with the full predictor calculations reproduced from the last panel of Figure 4. There is no difference between the two cases for the low wind speed period, September 20,02:00-07:00 hours. At other periods, significant differences between both curves can be observed.

Even when most of the wave energy is concentrated in swell

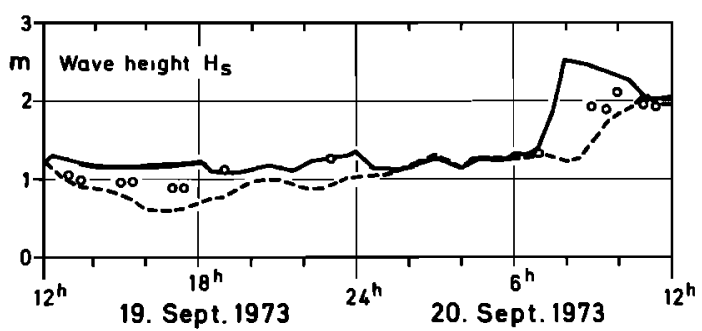

Fig. 17. Time series of significant wave height of hindcast A for station 9 for nonvanishing (solid line) and vanishing (dashed line) wind. The circles are measured values. 


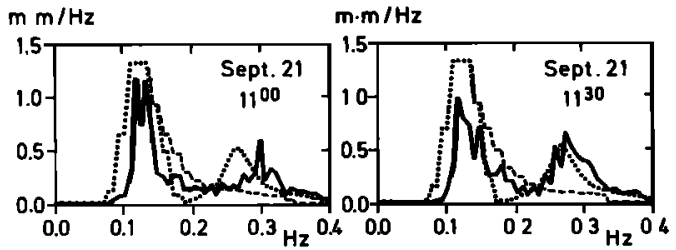

Fig. 18. Two spectra of hindcast B from station 9 for nonvanishing (dotted line) and vanishing (dashed line) wind. The solid line shows the measurement.

frequencies, so that the significant wave height for both cases does not differ significantly, the effect of the source function can generally be clearly seen in the wind-sea part of the spectrum. This is illustrated in Figure 18 by two successive spectra at station 9, taken from hindcast $B$.

As a third example, Figure 19 shows a comparison of the two hindcasts for the case of an onshore wind component producing considerable spectral development inside the test area. Here the propagation calculation without a source function differs appreciably from the complete prediction (reproduced from Figure 15).

\section{Conclusions}

Figures 20 and 21 show the linear regression line between observed and modeled peak frequency and significant wave height, respectively, for all periods of the three events for which measured values were available. The regression line is not restrained to go through the origin to show a possible systematic error for small values of wave height or peak frequency. As standard deviation from the $45^{\circ}$ line through the origin, we obtain for the peak frequency $\Delta f_{m}=0.02 \mathrm{~Hz}$ and for the significant wave height $\Delta H_{s}=0.30 \mathrm{~m}$.

As long as there does not exist a set of synoptic surface wave situations which is generally accepted for testing wave models, such an error statistic can of course only serve to show in a condensed manner the hindcast capability of a special wave model for the special data set of windfield and boundary conditions; it cannot be used to intercompare different wave models. In the present case, our data set is characterized by a relatively small prediction area and rather accurate knowledge of the wind field. Both factors tend to reduce the hindcast errors. On the other hand, the wind fields are chosen to be extremely nonstationary and in one case strongly inhomogeneous, thereby testing the model under rather extreme conditions.

From the inspection of our hindcasts we conclude that the

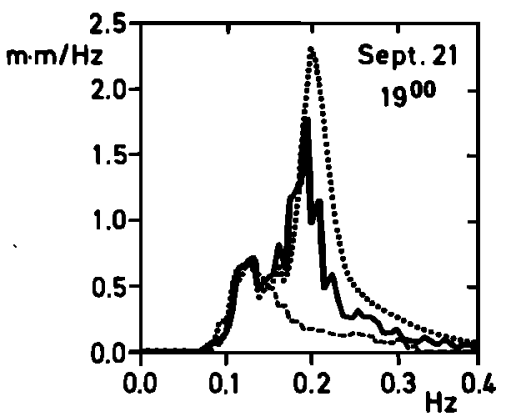

Fig. 19. A spectrum of hindcast $C$ from station 9 for nonvanishing (dotted line) and vanishing (dashed line) wind. The solid line shows the measurement.

\section{Correlation of peak frequency}

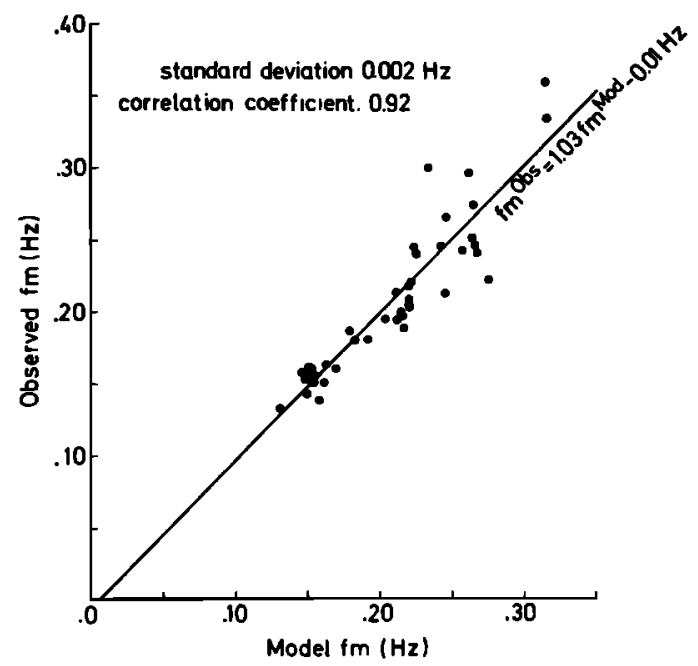

Fig. 20. Regression line between observed and hindcasted peak frequencies.

model is in the present form acceptable as a first-order deepwater surface wave prediction model. However, three important improvements should be made in the present version of the model: (1) inclusion of shallow water effects such as refraction and bottom dissipation, (2) incorporation of a directional relaxation time for turning winds, and (3) a physically more realistic algorithm for the transformation between wind sea and swell.

Item 1 can be improved in a straightforward manner, since the principles of refraction are known and investigations on dissipation have been made (for a review see Shemdin et al. [1977]). Item 2 can also be readily treated by the parametrical projection method, as outlined in paper $\mathrm{I}$. This requires the inclusion of additional directional parameters $\theta_{t}$ in the basic parameter set, the time evolution of these parameters then

\section{Correlation of significant waveheight $\mathrm{H}_{\mathrm{s}}$}

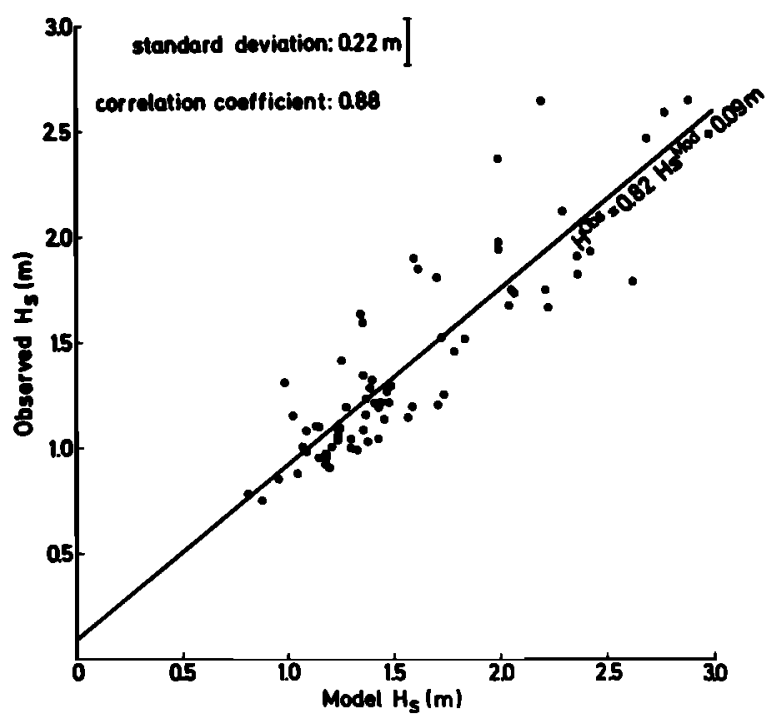

Fig. 21. Regression line between observed and hindcasted significant wave heights. 
being obtained by projecting the complete energy transport equation onto these parameters with appropriate projection functionals. The derivation of the corresponding source function in parameter space can be made by analysis of the directional measurements obtained during JONSWAP, or also by numerical evaluation of the nonlinear directional relaxation processes. Work on both aspects is in progress. Item 3 will require more detailed numerical integrations and subsequent parametrization of the nonlinear energy transfer between wind sea and swell in the critical transition region between the two regions of the spectrum.

Acknowledgments. The measurements used in this paper were made by the Deutsches Hydrographisches Institut, the Institute of Oceanographic Science, Wormley, the Institut für Meteorologie der Universität Hamburg, and the Koninklijk Nederlands Meteorologisch Instituut. Thanks are due to the many colleagues from these institutes who made these measurements available. We are grateful to $K$. Hasselmann for helpful advice. This work was supported by the Deutsche Forschungsgemeinschaft by means of the Sonderforschungsbereich 94, Meeresforschung, at the University of Hamburg.

\section{REFERENCES}

Barnett, T. P., C. H. Holland, Jr., and P. Yager, A general technique for wind wave prediction, with application to the South China Sea, contract N 62 306-68-C-0285, U.S. Nav. Oceanogr. Office, Washington, D. C., 1969.

Brümmer, B., D. Heinricy, L. Krlugermeyer, and D. Primm, The largescale weather features over the North Sea during the JONSWAP II experiment, Inst. der Fraunhofer Gesellschaft 24, Berichte des Inst. für Radiometeorologie und Maritime Meteorol. an der Univ. Hamburg, 1974.

Cartwright, D. E., The use of directional spectra in studying the output of a wave recorder on a moving ship, in Ocean Wave Spectra, Proceedings of a Conference, Prentice-Hall, Englewood Cliffs, N. J., 1961.

Ewing, J. A., B. A. Worthington, and T. J. Weare, A hindcast study of extreme wave conditions in the North Sea, J. Geophys. Res., 84, in press, 1979.

Günther, H., W. Rosenthal, T. J. Weare, B. A. Worthington, K. Hasselmann, and J. A. Ewing, A hybrid parameterical wave prediction model, J. Geophys. Res., 84, in press, 1979.

Hasselmann, K., On the application of recent energy balance investigations to numerical wave prediction, Turbulent fluxes through the sea surface, wave dynamics and prediction, in Air-Sea Interactions, vol. 1, NATO Conf. Ser., Plenum, New York, 1978.

Hasselmann, K., et al., Measurements of wind-wave growth and swell decay during the Joint North Sea Wave Project (JONSWAP), Er. gänzungsheft zur Deutschen Hydrographischen Z.. Reihe A, 8(12) 1973.

Hasselmann, K., D. B. Ross, P. Müller, and W. Sell, A parametric wave prediction model, J. Phys. Oceanogr., 6(2), 200-228, 1976.

Kinsman, B., Wind Waves: Their Generation and Propagation on the Ocean Surface, Prentice-Hall, Englewood Cliffs, N. J., 1965.

Shemdin, O., K. Hasselmann, V. Hsiao, and K. Herterich, Nonlinear and linear bottom interaction effects in shallow water, Proceeding of the NATO Symposium on Turbulent Fluxes Through the Sea Surface, Wave Dynamics and Prediction, North Atlantic Treaty Organ., Marseilles, Sept. 12-16, 1977.

(Received October 3, 1978;

revised January 19, 1979;

accepted February 15, 1979.) 